TITLE:

\title{
Magnetic properties of oxide glasses containing iron and rare- earth ions
}

AUTHOR(S):

Akamatsu, Hirofumi; Kawabata, Jun; Fujita, Koji; Murai, Shunsuke; Tanaka, Katsuhisa

CITATION:

Akamatsu, Hirofumi ... [et al]. Magnetic properties of oxide glasses

containing iron and rare-earth ions. PHYSICAL REVIEW B 2011, 84(14):

144408.

ISSUE DATE:

2011-10

URL:

http://hdl.handle.net/2433/161776

RIGHT:

(C)2011 American Physical Society 
PHYSICAL REVIEW B 84, 144408 (2011)

\title{
Magnetic properties of oxide glasses containing iron and rare-earth ions
}

\author{
Hirofumi Akamatsu, Jun Kawabata, Koji Fujita, Shunsuke Murai, and Katsuhisa Tanaka* \\ Department of Material Chemistry, Graduate School of Kyoto University, Nishikyo-ku, Kyoto 615-8510, Japan
}

(Received 6 June 2011; revised manuscript received 19 August 2011; published 5 October 2011)

\begin{abstract}
Measurements of fundamental magnetic properties including not only dc and ac susceptibilities but also magnetic aging effects have been performed for aluminoborate glasses with high concentrations of iron and rare-earth $R^{3+}$ ions $(R=\mathrm{Sm}, \mathrm{Gd}$, and $\mathrm{Tb})$ in order to give an insight into the magnetic structures and interactions in amorphous oxides containing both $3 d$ transition metal and $4 f$ rare-earth ions, which manifest magnetic interactions that differ from each other. We demonstrate that the antiferromagnetic interactions between iron and rare-earth ions as well as those between iron ions play a significant role for their magnetic properties, while those between rare-earth ions are of little importance. Most of the rare-earth ions remain paramagnetic even below the spin-freezing temperatures under the strong molecular field caused by the spin-glass freezing of the iron ions, as in the case of rare-earth garnet ferrites.
\end{abstract}

DOI: 10.1103/PhysRevB.84.144408

PACS number(s): 75.50.Kj, 75.10.Nr, 75.50.Lk

\section{INTRODUCTION}

Oxide glasses containing a high concentration of magnetic ions have attracted much interest as one of typical examples of insulating spin glass (SG) systems where longrange interactions such as Ruderman-Kittel-Kasuya-Yoshida interactions are not at work, in contrast to canonical SG. ${ }^{1}$ Instead, short-range antiferromagnetic (AFM) superexchange interactions via $2 p$ states of oxide ions are predominant in the magnetic oxide glasses, ${ }^{2-18}$ with some exceptions such as $\mathrm{Eu}^{2+}$-containing glasses, where ferromagnetic (FM) interactions prevail. ${ }^{19-23}$ In a system where magnetic moments are located at randomly distributed cations, the short-range AFM interactions inevitably bring about magnetic frustrations of geometrical origin. Consequently, SG phases appear at low temperatures due to the coexistence of randomness and frustration that are the key ingredients of SG.

Indeed, SG behavior has been observed in some oxide glasses containing a large amount of $3 d$ transition-metal (TM) ions such as $\mathrm{Mn}^{2+}, \mathrm{Fe}^{3+}$, and $\mathrm{Co}^{2+} \cdot{ }^{2-17}$ Those $3 d$ TM-containing glasses have negative Weiss temperatures, the amplitude of which is tens or hundreds of degrees Kelvin, and exhibit a paramagnetic (PM) to SG transition at several or tens of degrees Kelvin. On the other hand, most of the oxide glasses possessing $4 f$ rare-earth (RE) ions seldom show any magnetic transition down to $2 \mathrm{~K},{ }^{24,25}$ which can be easily accessed in magnetic measurements. This is because the AFM superexchange interactions between $4 f \mathrm{RE}$ ions are much smaller than those between $3 d$ TM ions. The $4 f$ electrons in the RE ions are localized at the inner shell, and, hence, the hopping of the $4 f$ electron to the $2 p$ orbitals of oxide ions is more difficult to occur compared with the $3 d$ electrons.

Although magnetic properties of oxide glasses with $3 d$ TM or $4 f \mathrm{RE}$ ions have been extensively studied as mentioned above, there are only a few reports on those of insulating glasses containing both $3 d$ TM and $4 f$ RE ions. ${ }^{5,16}$ As is well known, the magnetic interactions between $3 d$ TM and $4 f \mathrm{RE}$ ions lead to intriguing phenomena in crystalline oxides. For instance, in a series of RE garnet ferrites $R_{3} \mathrm{Fe}_{5} \mathrm{O}_{12}$, where $R$ is an $\mathrm{RE}$ ion, as the temperature is decreased, the magnetic moments of the $\mathrm{Fe}^{3+}$ ions first exhibit the ferrimagnetic order below Curie temperatures, and then the molecular-magnetic field derived by the ferrimagnetic $\mathrm{Fe}^{3+}$ moments renders the magnetic moments of $R^{3+}$ antiparallel to those of $\mathrm{Fe}^{3+} .{ }^{26}$ The magnetization due to the $\mathrm{Fe}^{3+}$ ions is perfectly canceled by that of the $R^{3+}$ ions at so-called compensation temperature. As another instance, a microscopic origin of the multiferroic properties in a perovskite orthoferrite $\mathrm{GdFeO}_{3}$ is considered to be a spin-exchange striction caused by the exchange interactions between the $\mathrm{Gd}^{3+}$ and $\mathrm{Fe}^{3+}$ ions. ${ }^{27}$

Thus, understanding magnetic interactions between magnetic moments of $3 d \mathrm{TM}$ and $4 f \mathrm{RE}$ ions in oxides is very important in view of both fundamentals and applications. Our previous report on the magnetic properties of amorphous thin films in binary $\mathrm{Fe}_{2} \mathrm{O}_{3}-R_{2} \mathrm{O}_{3}(R=\mathrm{La}, \mathrm{Gd}$, and $\mathrm{Tb})$ system has suggested that the magnetic structures of the films are explainable in terms of a superposition of SG behavior of the iron and PM behavior of the RE ions since the spin-freezing temperature is determined only by the concentration of iron ions and is independent of the kind and concentration of RE ions. ${ }^{16}$ However, a more cautious approach is necessary to discuss the magnetic structure and interactions because the form of thin films has rendered the qualitative interpretation of experimental data more complicated.

In this study, we examine the magnetic properties of bulk aluminoborate glasses containing iron and $R^{3+}$ ions $(R=\mathrm{Sm}$, $\mathrm{Gd}$, and $\mathrm{Tb}$ ) in high concentration. The main aim of this paper is to gain deep understanding of the magnetic interactions between the iron and RE ions and the magnetic structures in the oxide glass system. As a result, we conclude that the magnetic interactions between $\mathrm{Fe}$ and $\mathrm{RE}$ ions as well as those between $\mathrm{Fe}$ ions are important for the interpretation of the magnetic properties, and, at low temperatures, most of the RE ions remain to exhibit PM behavior experiencing the strong molecular field originating from the SG freezing of Fe ions.

The rest of the paper is organized as follows. In Sec. II, the experimental procedure is described. The valence state and local environment of iron in the present glasses are briefly discussed based on the results of Mössbauer spectroscopy in Sec. III A. In Sec. III B, the temperature dependence of dc magnetic susceptibility is reviewed based on the molecularfield model of two sublattice system, and the experimental data are analyzed using the theory to elucidate the magnetic 
interactions. In Sec. III C, we analyze the magnetic-field dependence of magnetization in detail. We present the temperature dependence of ac susceptibility, magnetic aging memory effects, and magnetic relaxation to confirm the SG nature of the present glasses in Secs. III D, III E, and III F, respectively. Finally, we summarize the experimental results and discussion and make a conclusion.

\section{EXPERIMENTAL PROCEDURE}

Glasses with nominal compositions (mol\%) of $x \mathrm{Fe}_{2} \mathrm{O}_{3} \cdot(20-$ $x) \mathrm{Ga}_{2} \mathrm{O}_{3} \cdot y R_{2} \mathrm{O}_{3} \cdot(20-y) \mathrm{La}_{2} \mathrm{O}_{3} \cdot 3 \mathrm{Al}_{2} \mathrm{O}_{3} \cdot 57 \mathrm{~B}_{2} \mathrm{O}_{3} \quad(x=0, \quad 15$, and 20, and $y=0,10$, and 20), where $R$ denotes the REs ( $\mathrm{Sm}, \mathrm{Gd}$, and $\mathrm{Tb}$ ), were prepared from reagent-grade $\mathrm{Fe}_{2} \mathrm{O}_{3}$, $\mathrm{Ga}_{2} \mathrm{O}_{3}, \mathrm{La}_{2} \mathrm{O}_{3}, \mathrm{Sm}_{2} \mathrm{O}_{3}, \mathrm{Gd}_{2} \mathrm{O}_{3}, \mathrm{~Tb}_{4} \mathrm{O}_{7}, \mathrm{Al}_{2} \mathrm{O}_{3}$, and $\mathrm{B}_{2} \mathrm{O}_{3}$ powders via conventional melt-quenching method. The glass compositions and their notations are listed in Table I. The starting raw materials were weighed to obtain the prescribed compositions and mixed thoroughly in an alumina mortar. The mixture was then melted in an alumina crucible for 20 min at $1300^{\circ} \mathrm{C}$ in air. The melt was poured onto a stainless steel plate and cooled in air. The amorphous nature of the samples was confirmed by using x-ray diffraction with $\mathrm{Cu} \mathrm{K} \alpha$ radiation.

${ }^{57} \mathrm{Fe}$ Mössbauer spectroscopy was carried out at room temperature employing ${ }^{57} \mathrm{Co}$ in metallic $\mathrm{Rh}$ as a $\gamma$-ray source to estimate valence state and coordination environment for iron ion in the glasses. The velocity scale was calibrated by using a spectrum of $\alpha$-Fe foil measured at room temperature. Isomer shifts (IS) were evaluated with respect to $\alpha$-Fe. Magnetic properties were measured by using a superconducting quantum interference device magnetometer (Quantum Design, model MPMS-XL).

\section{RESULTS AND DISCUSSION}

\section{A. ${ }^{57} \mathrm{Fe}$ Mössbauer spectroscopy}

Figure 1 shows the Mössbauer spectrum for the $20 \mathrm{Fe}$ glass at room temperature. The spectrum is a superposition of two PM doublets attributed to $\mathrm{Fe}^{2+}$ and $\mathrm{Fe}^{3+}$ ions. It is found that the $\mathrm{Fe}^{2+}$ and $\mathrm{Fe}^{3+}$ ions are $\mathrm{PM}$ at room temperature in the present glass. Because of the asymmetry and broadness of the absorption spectra, the experimental spectra were fitted with broadened Lorentzian doublets consisting of multiple symmetric Lorentzian doublets with a full width of half

TABLE I. Glass compositions and their notations.

\begin{tabular}{lc}
\hline \hline Notation & Composition (mol\%) \\
\hline $15 \mathrm{Fe}$ & $15 \mathrm{Fe}_{2} \mathrm{O}_{3} \cdot 5 \mathrm{Ga}_{2} \mathrm{O}_{3} \cdot 20 \mathrm{La}_{2} \mathrm{O}_{3} \cdot 3 \mathrm{Al}_{2} \mathrm{O}_{3} \cdot 57 \mathrm{~B}_{2} \mathrm{O}_{3}$ \\
$20 \mathrm{Fe}$ & $20 \mathrm{Fe}_{2} \mathrm{O}_{3} \cdot 20 \mathrm{La}_{2} \mathrm{O}_{3} \cdot 3 \mathrm{Al}_{2} \mathrm{O}_{3} \cdot 57 \mathrm{~B}_{2} \mathrm{O}_{3}$ \\
$20 \mathrm{Fe} 10 \mathrm{Sm}$ & $20 \mathrm{Fe}_{2} \mathrm{O}_{3} \cdot 10 \mathrm{Sm}_{2} \mathrm{O}_{3} \cdot 10 \mathrm{La}_{2} \mathrm{O}_{3} \cdot 3 \mathrm{Al}_{2} \mathrm{O}_{3} \cdot 57 \mathrm{~B}_{2} \mathrm{O}_{3}$ \\
$20 \mathrm{Fe} 20 \mathrm{Sm}$ & $20 \mathrm{Fe}_{2} \mathrm{O}_{3} \cdot 20 \mathrm{Sm}_{2} \mathrm{O}_{3} \cdot 3 \mathrm{Al}_{2} \mathrm{O}_{3} \cdot 57 \mathrm{~B}_{2} \mathrm{O}_{3}$ \\
$20 \mathrm{Fe} 10 \mathrm{Gd}$ & $20 \mathrm{Fe}_{2} \mathrm{O}_{3} \cdot 10 \mathrm{Gd}_{2} \mathrm{O}_{3} \cdot 10 \mathrm{La}_{2} \mathrm{O}_{3} \cdot 3 \mathrm{Al}_{2} \mathrm{O}_{3} \cdot 57 \mathrm{~B}_{2} \mathrm{O}_{3}$ \\
$20 \mathrm{Fe} 20 \mathrm{Gd}$ & $20 \mathrm{Fe}_{2} \mathrm{O}_{3} \cdot 20 \mathrm{Gd}_{2} \mathrm{O}_{3} \cdot 3 \mathrm{Al}_{2} \mathrm{O}_{3} \cdot 57 \mathrm{~B}_{2} \mathrm{O}_{3}$ \\
$20 \mathrm{Gd}$ & $20 \mathrm{Ga}_{2} \mathrm{O}_{3} \cdot 20 \mathrm{Gd}_{2} \mathrm{O}_{3} \cdot 3 \mathrm{Al}_{2} \mathrm{O}_{3} \cdot 57 \mathrm{~B}_{2} \mathrm{O}_{3}$ \\
$20 \mathrm{Fe} 10 \mathrm{~Tb}$ & $20 \mathrm{Fe}_{2} \mathrm{O}_{3} \cdot 10 \mathrm{~Tb}_{2} \mathrm{O}_{3} \cdot 10 \mathrm{La}_{2} \mathrm{O}_{3} \cdot 3 \mathrm{Al}_{2} \mathrm{O}_{3} \cdot 57 \mathrm{~B}_{2} \mathrm{O}_{3}$ \\
$20 \mathrm{Fe} 20 \mathrm{~Tb}$ & $20 \mathrm{Fe}_{2} \mathrm{O}_{3} \cdot 20 \mathrm{~Tb}_{2} \mathrm{O}_{3} \cdot 3 \mathrm{Al}_{2} \mathrm{O}_{3} \cdot 57 \mathrm{~B}_{2} \mathrm{O}_{3}$ \\
$20 \mathrm{~Tb}$ & $20 \mathrm{Ga}_{2} \mathrm{O}_{3} \cdot 20 \mathrm{~Tb}_{2} \mathrm{O}_{3} \cdot 3 \mathrm{Al}_{2} \mathrm{O}_{3} \cdot 57 \mathrm{~B}_{2} \mathrm{O}_{3}$ \\
\hline \hline
\end{tabular}

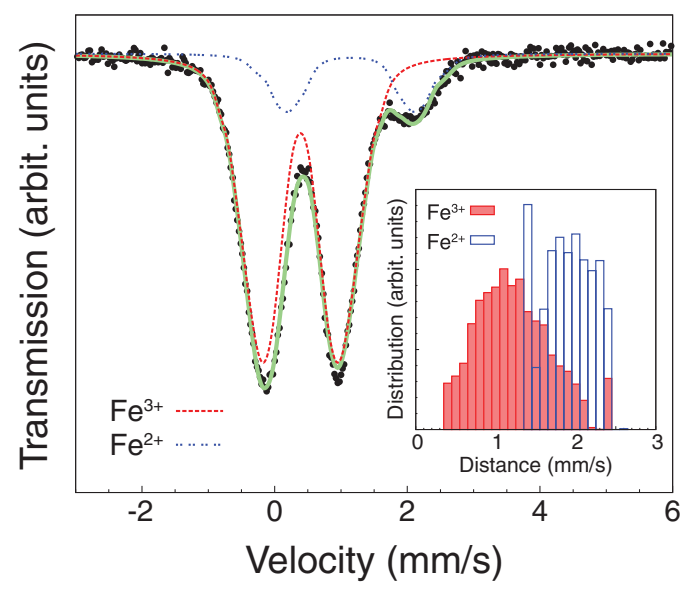

FIG. 1. (Color online) ${ }^{57} \mathrm{Fe}$ Mössbauer spectrum for $20 \mathrm{Fe}$ glass at room temperature. Experimental and computed absorption spectra are indicated by dots and the solid line, respectively. Calculated spectra attributed to $\mathrm{Fe}^{3+}$ and $\mathrm{Fe}^{2+}$ are represented by dashed lines. The inset shows distribution of distance between the peaks of doublets.

maximum of $0.25 \mathrm{~mm} / \mathrm{s}$ equal to the natural width of iron foil. ${ }^{28}$ The individual Lorentzian doublets have the identical middle point, i.e., IS, but with the varied separation between the peaks. The calculated spectrum is illustrated in Fig. 1. The inset of Fig. 1 depicts distribution of the peak-to-peak separations for $\mathrm{Fe}^{3+}$ and $\mathrm{Fe}^{2+}$. It is reasonable to regard the average values of the peak separations as quadrupole splitting (QS), and the absorption area ratio of the $\mathrm{Fe}^{3+}$ and $\mathrm{Fe}^{2+}$ ions obtained by the present fitting method is reliable, as pointed out by Virgo and Mysen. ${ }^{28}$ The fraction of the absorption area of $\mathrm{Fe}^{3+}$ ions in the total area is $85 \%$ for the $20 \mathrm{Fe}$ glass, and the IS and QS values are 0.39 and $1.23 \mathrm{~mm} / \mathrm{s}$ for the $\mathrm{Fe}^{3+}$ ion and 1.15 and $1.97 \mathrm{~mm} / \mathrm{s}$ for the $\mathrm{Fe}^{2+}$ ion, respectively. Similar IS and QS values and absorption area ratio of the $\mathrm{Fe}^{3+}$ and $\mathrm{Fe}^{2+}$ ions were obtained for the other glasses as shown in Table II. Such IS and QS values indicate that both of the $\mathrm{Fe}^{2+}$ and $\mathrm{Fe}^{3+}$ ions mainly occupy octahedral sites surrounded by $\mathrm{O}^{2-}$ ions in the glasses.

TABLE II. Mössbauer parameters obtained by fitting calculated curves to the experimental spectra. The area ratio of the $\mathrm{Fe}^{3+}$ absorption relative to the total absorption, $A_{\mathrm{Fe}^{3+}} / A_{\text {total }}$, is also shown.

\begin{tabular}{lcccccc}
\hline \hline \multirow{2}{*}{ Sample } & \multicolumn{2}{c}{$\mathrm{Fe}^{3+}$} & & \multicolumn{2}{c}{$\mathrm{Fe}^{2+}$} & \\
\cline { 2 - 3 } & $\begin{array}{c}\mathrm{IS} \\
(\mathrm{mm} / \mathrm{s})\end{array}$ & $\begin{array}{c}\mathrm{QS} \\
(\mathrm{mm} / \mathrm{s})\end{array}$ & & $\begin{array}{c}\mathrm{IS} \\
(\mathrm{mm} / \mathrm{s})\end{array}$ & $\begin{array}{c}\mathrm{QS} \\
(\mathrm{mm} / \mathrm{s})\end{array}$ & $A_{\mathrm{Fe}^{3+}} / A_{\text {total }}$ \\
\hline $15 \mathrm{Fe}$ & 0.39 & 1.22 & & 1.12 & 2.03 & 0.84 \\
$20 \mathrm{Fe}$ & 0.39 & 1.23 & & 1.15 & 1.97 & 0.85 \\
20Fe10Sm & 0.39 & 1.24 & & 1.13 & 1.99 & 0.83 \\
20Fe20Sm & 0.39 & 1.24 & & 1.15 & 2.00 & 0.83 \\
20Fe10Gd & 0.39 & 1.24 & & 1.14 & 2.01 & 0.82 \\
20Fe20Gd & 0.40 & 1.24 & & 1.14 & 2.01 & 0.83 \\
20Fe10Tb & 0.39 & 1.23 & & 1.15 & 2.01 & 0.84 \\
20Fe20Tb & 0.39 & 1.24 & 1.14 & 2.01 & 0.84 \\
\hline \hline
\end{tabular}




\section{B. Temperature dependence of dc magnetic susceptibility}

\section{Molecular-field theory of two sublattice model}

Here, we begin by considering the temperature dependence of magnetic susceptibility for the present glasses based on the molecular-field theory of two-sublattice system. The magnetic system for the present glasses under an external magnetic field, $h$, can be modeled by the following Heisenberg Hamiltonian:

$$
\begin{aligned}
\mathcal{H}= & -\sum_{i>j} 2 J_{i j} \mathbf{S}_{i} \cdot \mathbf{S}_{j}-g \mu_{B} h \sum_{i} \mathbf{S}_{i, z} \\
= & -\sum_{i>j}^{\mathrm{Fe}} 2 J_{\mathrm{Fe}^{i} \mathrm{Fe}^{j}} \mathbf{S}_{\mathrm{Fe}^{i}} \cdot \mathbf{S}_{\mathrm{Fe}^{j}}-\sum_{i>j}^{R} 2 J_{R^{i} R^{j}} \mathbf{S}_{R^{i}} \cdot \mathbf{S}_{R^{j}} \\
& -\sum_{i, j}^{\mathrm{Fe}, R} 2 J_{\mathrm{Fe}^{i} R^{j}} \mathbf{S}_{\mathrm{Fe}^{i}} \cdot \mathbf{S}_{R^{j}}-g \mu_{B} h \sum_{i} \mathbf{S}_{i, z},
\end{aligned}
$$

where $R$ is an RE ion, $\mathbf{S}_{i}$ is the spin operator at the $i$ site, $J_{i j}$ is the exchange constant of the magnetic interaction between the $i$ and $j$ sites, $g$ is the Lande's $g$ factor, and $\mu_{B}$ is the Bohr magneton. Therefore, the magnetic fields affecting the Fe and RE ion are

$$
h_{\mathrm{Fe}}=\gamma_{\mathrm{FeFe}} M_{\mathrm{Fe}}+\gamma_{\mathrm{Fe} R} M_{R}+h
$$

and

$$
h_{R}=\gamma_{R R} M_{R}+\gamma_{R \mathrm{Fe}} M_{\mathrm{Fe}}+h,
$$

respectively, where $M_{A}$ is the magnetization of $A$ ions. Here, although $M_{\mathrm{Fe}}$ is the average value of magnetization of $\mathrm{Fe}^{2+}$ and $\mathrm{Fe}^{3+}$ ions, the $M_{\mathrm{Fe}}$ value is attributed mainly to $\mathrm{Fe}^{3+}$ ions because about $85 \%$ of the total $\mathrm{Fe}$ ions are present as the trivalent state in the present glasses. The molecular-field coefficient, $\gamma_{A B}$, is represented as:

$$
\gamma_{A B}=\frac{2 z_{A B} J_{A B}}{N_{B} g^{2} \mu_{B}^{2}},
$$

where $N_{B}$ is the number of magnetic ions per a mole of formula unit, $J_{A B}$ is the mean exchange constant of the magnetic interaction between the $A$ and $B$ sites $\left(J_{A B}=J_{B A}\right)$, and $z_{A B}$ is the mean number of $B$ ions neighboring an $A$ ion. Consequently, the magnetization of the $A$ ions is self-consistently determined by the following equation:

$$
M_{A}=M_{A 0} B_{S}\left(h_{A}\right),
$$

where $B_{s}$ is a Brillouin function and $M_{A 0}$ is the magnetization of the $A$ ions at $0 \mathrm{~K}$. The temperature dependence of magnetic susceptibility, $\chi(T)$, at high temperatures is approximated by the following equation:

$$
\chi(T)=\frac{C_{\mathrm{Fe}}+C_{R}}{T-\theta_{W}} .
$$

Here, the Curie constant of the $A$ ions, $C_{A}$, is represented by

$$
C_{A}=\frac{N_{A} m_{A}^{2} \mu_{B}^{2}}{3 k_{B}},
$$

where $m_{A}$ is the effective number of Bohr magnetons for the $A$ ions and $k_{B}$ is the Boltzmann constant. The Weiss temperature, $\theta_{W}$, is described by

$$
\theta_{W}=\frac{C_{\mathrm{Fe}}^{2} \gamma_{\mathrm{FeFe}}+C_{R}^{2} \gamma_{R R}+C_{\mathrm{Fe}} C_{R}\left(\gamma_{\mathrm{Fe} R}+\gamma_{R \mathrm{Fe}}\right)}{C_{\mathrm{Fe}}+C_{R}} .
$$

When $\mathrm{Fe}$ ion is absent in the glass, i.e., $C_{\mathrm{Fe}}=0, \theta_{W}^{R}=$ $C_{R} \gamma_{R R}$. In addition, when magnetic RE ion is absent in the glass, i.e., $C_{R}=0, \theta_{W}^{\mathrm{Fe}}=C_{\mathrm{Fe}} \gamma_{\mathrm{FeFe}}$. Here, we define the effective Weiss temperature by

$$
\theta_{W}^{\mathrm{Fe} R}=\sqrt{C_{\mathrm{Fe}} C_{R}} \frac{\gamma_{\mathrm{Fe} R}+\gamma_{R \mathrm{Fe}}}{2},
$$

which is a measure of the strength of magnetic interaction between the Fe and RE ions. Consequently, $\theta_{W}$ can be rewritten as follows:

$$
\theta_{W}=\frac{C_{\mathrm{Fe}} \theta_{W}^{\mathrm{Fe}}+C_{R} \theta_{W}^{R}+2 \sqrt{C_{\mathrm{Fe}} C_{R}} \theta_{W}^{\mathrm{Fe} R}}{C_{\mathrm{Fe}}+C_{R}} .
$$

\section{Experimental data}

Figure 2(a) displays the dc magnetic susceptibility for $15 \mathrm{Fe}$ and $20 \mathrm{Fe}$ glasses. Both zero-field-cooling (ZFC) and field-cooling (FC) processes were carried out under a magnetic field of $50 \mathrm{Oe}$. The $15 \mathrm{Fe}$ glass is $\mathrm{PM}$ down to $2 \mathrm{~K}$; the temperature dependencies of dc susceptibilities for the ZFC and FC processes, $\chi_{\mathrm{ZFC}}(T)$ and $\chi_{\mathrm{FC}}(T)$, respectively, increase as the temperature is decreased and merge with each other. On the other hand, for the $20 \mathrm{Fe}$ glass, $\chi_{\mathrm{ZFC}}(T)$ exhibits a peak at $3.6 \mathrm{~K}$ and $\chi_{\mathrm{FC}}(T)$ becomes higher than $\chi_{\mathrm{ZFC}}(T)$ at lower temperatures, indicating SG behavior. A close look at
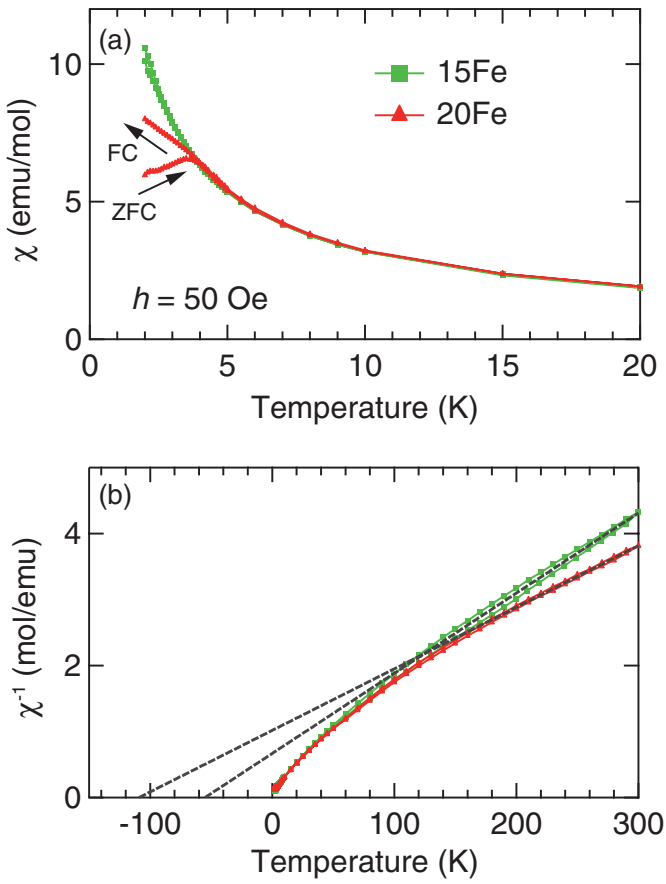

FIG. 2. (Color online) Temperature dependence of (a) dc magnetic susceptibility and (b) its reciprocal for $15 \mathrm{Fe}$ and $20 \mathrm{Fe}$ glasses. The dashed lines represent the best fit of Eq. (6) at high temperatures. 
the $\chi(T)$ curve reveals that $\chi_{\mathrm{FC}}(T)$ deviates from $\chi_{\mathrm{ZFC}}(T)$ below $4.5 \mathrm{~K}$, which is higher than the peak temperature, i.e., $3.6 \mathrm{~K}$, and increases monotonously at lower temperatures. This differs slightly from the behavior of canonical SGs such as $C u \mathrm{Mn}^{29}$ and oxide glasses such as iron tellurite and phosphate glasses, ${ }^{11,17}$ where $\chi_{\mathrm{FC}}(T)$ deviates from $\chi_{\mathrm{ZFC}}(T)$ and becomes almost constant just below the peak temperature. This implies that some of the magnetic moments of Fe ions form magnetic clusters in the present glass and the larger clusters start to freeze at $4.5 \mathrm{~K}$ with lowering temperature. Hereafter, the spin-freezing temperature, $T_{f}$, is defined as the temperature at which $\chi_{\mathrm{ZFC}}(T)$ deviates from $\chi_{\mathrm{FC}}(T)$ as the temperature is decreased.

The temperature dependence of the inverse susceptibility, $\chi^{-1}(T)$, is shown in Fig. 2(b). The dashed lines represent the best fits of Eq. (6) at high temperatures. The analyses yield $m_{\mathrm{Fe}}=4.7$ and $\theta_{W}^{\mathrm{Fe}}=-55 \mathrm{~K}$ for the $15 \mathrm{Fe}$ glass and $m_{\mathrm{Fe}}=4.6$ and $\theta_{W}^{\mathrm{Fe}}=-109 \mathrm{~K}$ for the $20 \mathrm{Fe}$ glass. The negative values of $\theta_{W}^{\mathrm{Fe}}$ mean that the AFM interaction is dominant among the $\mathrm{Fe}$ ions. The values of $m_{\mathrm{Fe}}$ are slightly smaller than those expected from the valence states of $\mathrm{Fe}$ ions in the present glasses as well as the spin-only value of $\mathrm{Fe}^{2+}$ and $\mathrm{Fe}^{3+}$ ions, i.e., 4.8 and 5.9, respectively. This implies the presence of short-range AFM correlations between the magnetic moments of the Fe ions at high temperatures near room temperature.

Figures 3(a) and 3(b) show the $\chi(T)$ curves for 20Fe10Gd, $20 \mathrm{Fe} 20 \mathrm{Gd}$, and $20 \mathrm{Gd}$ glasses and $\chi^{-1}(T)$ for $20 \mathrm{Fe} 20 \mathrm{Gd}$ and $20 \mathrm{Gd}$ glasses. For the $20 \mathrm{Gd}$ glass, $\chi_{\mathrm{ZFC}}(T)$ coincides with $\chi_{\mathrm{FC}}(T)$ and increases with lowing temperature, indicating that the present glass is PM down to $2 \mathrm{~K}$. Analysis of the $\chi^{-1}(T)$
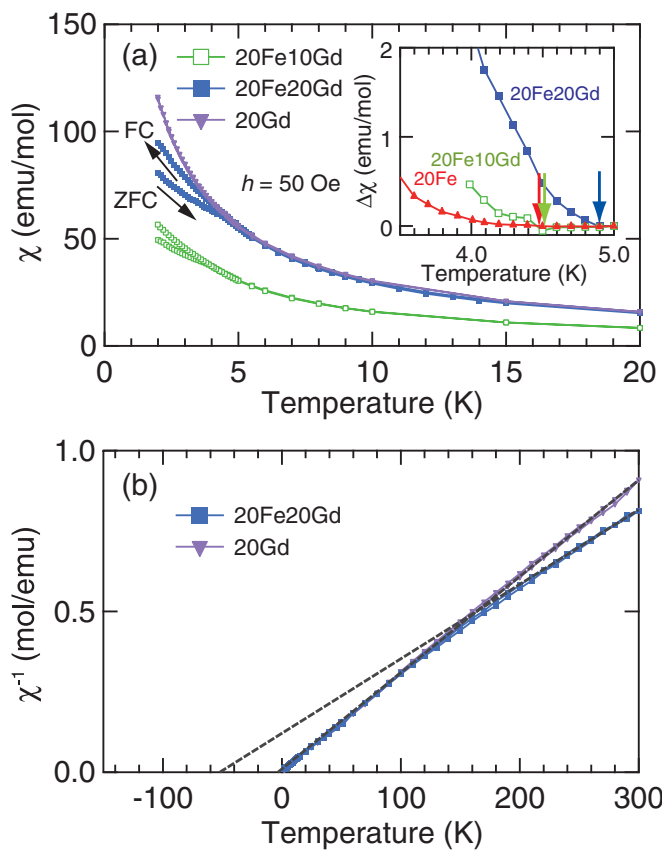

FIG. 3. (Color online) (a) Temperature dependence of dc magnetic susceptibility for $20 \mathrm{Fe} 10 \mathrm{Gd}, 20 \mathrm{Fe} 20 \mathrm{Gd}$, and 20Gd glasses, and (b) its reciprocal for $20 \mathrm{Fe} 20 \mathrm{Gd}$ and $20 \mathrm{Gd}$ glasses. The difference in dc susceptibility between the field-cooling and zero-field-cooling processes, $\Delta \chi=\chi_{\mathrm{FC}}-\chi_{\mathrm{ZFC}}$, is plotted as a function of temperature for the $20 \mathrm{Fe}, 20 \mathrm{Fe} 10 \mathrm{Gd}$, and $20 \mathrm{Fe} 20 \mathrm{Gd}$ glasses in the inset of (a). The arrows indicate the spin-freezing temperatures. by Eq. (6) yields $m_{\mathrm{Gd}}=8.2$ and $\theta_{W}^{\mathrm{Gd}}=-3.2 \mathrm{~K}$ for the $20 \mathrm{Gd}$ glass as shown in Fig. 3(b). The negative value of $\theta_{W}^{\mathrm{Gd}}$ implies that the AFM interaction is dominant among the $\mathrm{Gd}$ ions. The value of $m_{\mathrm{Gd}}$ is comparable with the theoretical value of a free $\mathrm{Gd}^{3+}$ ion $\left({ }^{8} \mathrm{~S}_{7 / 2}\right)$, i.e., 7.94 .

On the other hand, $\chi_{\mathrm{ZFC}}(T)$ also exhibits no maximum for the $20 \mathrm{Fe} 10 \mathrm{Gd}$ and $20 \mathrm{Fe} 20 \mathrm{Gd}$ glasses, but $\chi_{\mathrm{FC}}(T)$ deviates from $\chi_{\mathrm{ZFC}}(T)$ below $T_{f}=4.5$ and $4.9 \mathrm{~K}$, respectively, as shown in the inset of Fig. 3(a), which illustrates the difference in dc susceptibility between the FC and ZFC processes, $\Delta \chi(T)=$ $\chi_{\mathrm{FC}}(T)-\chi_{\mathrm{ZFC}}(T)$ for the $20 \mathrm{Fe}, 20 \mathrm{Fe} 10 \mathrm{Gd}$, and $20 \mathrm{Fe} 20 \mathrm{Gd}$ glasses.

The $\chi(T)$ curves seem to be simply explained in terms of a superposition of the PM behavior of the $\mathrm{Gd}$ ions and the SG behavior of the $\mathrm{Fe}$ ions. However, the magnetic interactions between the $\mathrm{Fe}$ and $\mathrm{Gd}$ ions are found to play a significant role in their magnetic properties as follows: first, $\chi(T)$ for the $20 \mathrm{Fe} 20 \mathrm{Gd}$ glass is smaller than that for the 20Gd glass, although the 20Fe20Gd glass has additional Fe magnetic moments compared to the 20Gd glass. The magnetic susceptibility of the Gd ions is suppressed by the AFM interaction between the Fe and Gd ions. Second, $T_{f}$ becomes higher with an increase in the Gd contents, implying that the magnetic interactions between the $\mathrm{Fe}$ and $\mathrm{Gd}$ ions have some effect on the SG freezing. Here, it should be mentioned, however, that it is difficult to see whether the magnetic moments of the Gd ions exhibit the SG freezing. Although it cannot be excluded that some of the magnetic moments of $\mathrm{Gd}$ ions may freeze into a SG state at the site where the molecular field formed by the iron ions is strong, it is inferred that Gd ions exhibit PM behavior for the most part even below $T_{f}$ on the basis of the fact that both $\chi_{\mathrm{ZFC}}(T)$ and $\chi_{\mathrm{FC}}(T)$ increase with lowering temperature below $T_{f}$.

The analysis of $\chi^{-1}(T)$ at high temperatures by using Eq. (6) gives $m_{\mathrm{Fe}, \mathrm{Gd}}=6.6$ and $\theta_{W}=-52 \mathrm{~K}$ for the $20 \mathrm{Fe} 20 \mathrm{Gd}$ glass. The $m_{\mathrm{Fe}, \mathrm{Gd}}$ value is comparable with the mean values of $m_{\mathrm{Fe}}$ and $m_{\mathrm{Gd}}$ obtained for the the 20Fe and 20Gd glasses, respectively. Using Eq. (10) and the values of $C_{A}$ and $\theta_{W}^{A}$ for $A=\mathrm{Fe}$ and $\mathrm{Gd}$ obtained by the analyses of the $\chi^{-1}(T)$ for the $20 \mathrm{Fe}$ and $20 \mathrm{Gd}$ glasses, respectively, we obtain $\theta_{W}^{\mathrm{FeGd}}=-27 \mathrm{~K}$. The negative value of $\theta_{W}^{\mathrm{FeGd}}$ indicates the AFM interactions between the Fe and $\mathrm{Gd}$ ions. Comparing $\theta_{W}^{\mathrm{FeGd}}$ with $\theta_{W}^{\mathrm{Fe}}$ and $\theta_{W}^{\mathrm{Gd}}$, it is found that the magnetic interaction between the $\mathrm{Fe}$ and $\mathrm{Gd}$ ions is weaker and stronger than those between the Fe ions and between the Gd ions, respectively.

The magnetic behavior of $20 \mathrm{~Tb}, 20 \mathrm{Fe} 10 \mathrm{~Tb}$, and $20 \mathrm{Fe} 20 \mathrm{~Tb}$ glasses is qualitatively similar to that of the $20 \mathrm{Gd}, 20 \mathrm{Fe} 10 \mathrm{Gd}$, 20Fe20Gd glasses, respectively, as shown in Fig. 4(a). The fit of Eq. (6) to $\chi^{-1}(T)$ yields $m_{\mathrm{Tb}}=10.1$ and $\theta_{W}^{\mathrm{Tb}}=-6.8 \mathrm{~K}$ for the $20 \mathrm{~Tb}$ glass (data are not shown). The $m_{\mathrm{Tb}}$ value for the $20 \mathrm{~Tb}$ glass is comparable with that expected from the ground state of a free $\mathrm{Tb}^{3+}$ ion $\left({ }^{7} \mathrm{~F}_{6}\right)$, i.e., 9.72 .

The $\chi(T)$ curves are shown for $20 \mathrm{Fe}$ and $20 \mathrm{Fe} 20 \mathrm{Sm}$ glasses in Fig. 4(b). It is found that $\chi(T)$ for the $20 \mathrm{Fe} 20 \mathrm{Sm}$ glass is slightly larger than that for the $20 \mathrm{Fe}$ glass. The magnetic moments of the ground state of $\mathrm{Sm}^{2+}\left({ }^{7} \mathrm{~F}_{0}\right)$ and $\mathrm{Sm}^{3+}\left({ }^{6} \mathrm{H}_{5 / 2}\right)$ are 0 and $0.85 \mu_{B}$, respectively, and these ions actually have the larger magnetic moments due to the contribution of van Vleck paramagnetism. The magnetic moment of $\mathrm{Sm}$ ions 

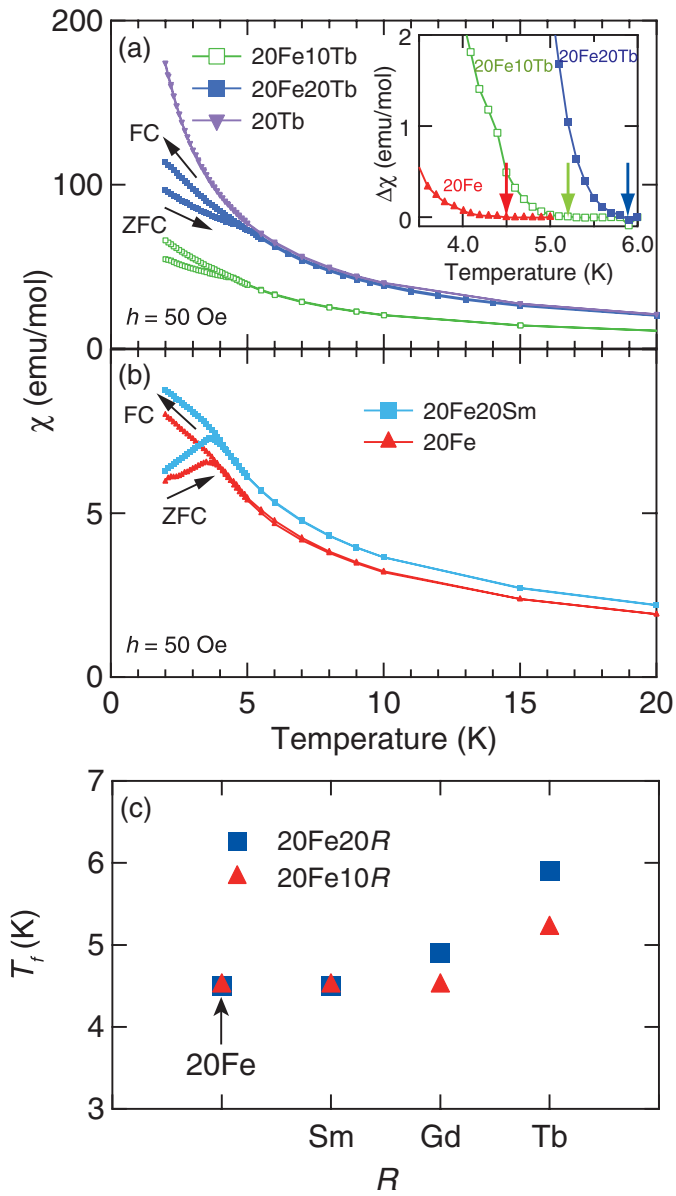

FIG. 4. (Color online) Temperature dependence of dc magnetic susceptibility (a) for $20 \mathrm{Fe} 10 \mathrm{~Tb}, 20 \mathrm{Fe} 20 \mathrm{~Tb}$, and $20 \mathrm{~Tb}$ glasses and (b) for $20 \mathrm{Fe}$ and $20 \mathrm{Fe} 20 \mathrm{Sm}$ glasses. The difference in dc susceptibility between the field-cooling and zero-field-cooling processes, $\Delta \chi=\chi_{\mathrm{FC}}-\chi_{\mathrm{ZFC}}$, is plotted as a function of temperature for the $20 \mathrm{Fe}, 20 \mathrm{Fe} 10 \mathrm{~Tb}$, and $20 \mathrm{Fe} 20 \mathrm{~Tb}$ glasses in the inset of (a). The arrows indicate the spin-freezing temperatures. (c) Spin-freezing temperatures for $20 \mathrm{Fe} 10 R$ and $20 \mathrm{Fe} 20 R(R=\mathrm{Sm}, \mathrm{Gd}$, and $\mathrm{Tb})$ and $20 \mathrm{Fe}$ glasses.

is nonetheless much smaller than those of $\mathrm{Tb}^{3+}$ and $\mathrm{Gd}^{3+}$. Therefore, the magnetic behavior of the 20Fe20Sm glass is similar to that of the $20 \mathrm{Fe}$ glass. In particular, the $T_{f}$ of the $20 \mathrm{Fe} 20 \mathrm{Sm}$ glass is found to be almost the same as that of the 20Fe glass.

Figure 4(c) illustrates the variation of $T_{f}$ with the kind of $\mathrm{RE}$ ion and its concentration. It is found that $T_{f}$ increases as the magnetic moment of RE ion is larger and as the content of $\mathrm{RE}$ ion is higher. In RE garnet ferrites, the weak dependence of Curie temperature on the sort of RE ions is also observed. ${ }^{26}$

\section{Magnetic-field dependence of magnetization}

The magnetic-field dependence of magnetization has been examined in order to discuss in more detail the magnetic interactions among the $\mathrm{Fe}$ and $\mathrm{RE}$ ions. The measurements were performed at $2 \mathrm{~K}$ in the magnetic-field range from 0 to 50000 Oe.
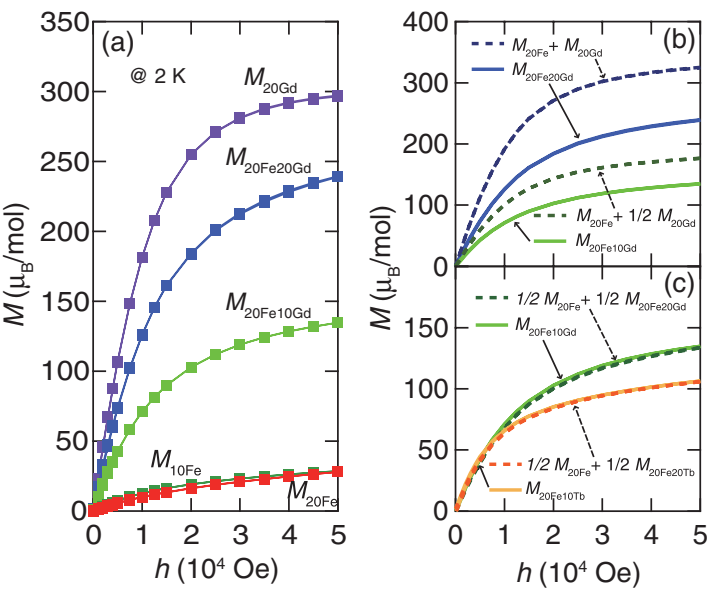

(d)
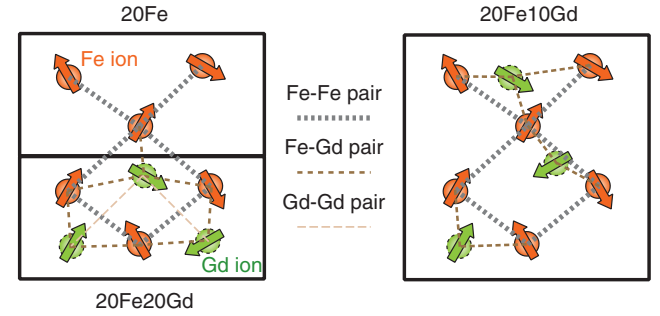

FIG. 5. (Color online) (a) The magnetic-field dependence of magnetization at $2 \mathrm{~K}$ for $20 \mathrm{Gd}, 20 \mathrm{Fe} 20 \mathrm{Gd}, 20 \mathrm{Fe} 10 \mathrm{Gd}, 20 \mathrm{Fe}$, and $10 \mathrm{Fe}$ glasses, $M_{20 \mathrm{Gd}}, M_{20 \mathrm{Fe} 20 \mathrm{Gd}}, M_{20 \mathrm{Fe} 10 \mathrm{Gd}}, M_{20 \mathrm{Fe}}$, and $M_{10 \mathrm{Fe}}$, respectively. Plotted against magnetic field are (b) $M_{20 \mathrm{Fe} 20 \mathrm{Gd}}, M_{20 \mathrm{Fe}}+$ $M_{20 \mathrm{Gd}}, M_{20 \mathrm{Fe} 10 \mathrm{Gd}}$, and $M_{20 \mathrm{Fe}}+\frac{1}{2} M_{20 \mathrm{Gd}}$ and (c) $M_{20 \mathrm{Fe} 10 \mathrm{Gd}}, \frac{1}{2} M_{20 \mathrm{Fe}}+$ $\frac{1}{2} M_{20 \mathrm{Fe} 20 \mathrm{Gd}}, M_{20 \mathrm{Fe} 10 \mathrm{~Tb}}$, and $\frac{1}{2} M_{20 \mathrm{Fe}}+\frac{1}{2} M_{20 \mathrm{Fe} 20 \mathrm{~Tb}}$. (d) Schematic models for $M_{20 \mathrm{Fe} 10 \mathrm{Gd}}$ and $\frac{1}{2} M_{20 \mathrm{Fe} 20 \mathrm{Gd}}+\frac{1}{2} M_{20 \mathrm{Fe}}$ are illustrated in the right and left panels, respectively.

Figure 5(a) shows the field dependence of magnetization for $20 \mathrm{Gd}, 20 \mathrm{Fe} 20 \mathrm{Gd}, 20 \mathrm{Fe} 10 \mathrm{Gd}, 20 \mathrm{Fe}$, and $10 \mathrm{Fe}$ glasses, $M_{20 \mathrm{Gd}}, M_{20 \mathrm{Fe} 20 \mathrm{Gd}}, M_{20 \mathrm{Fe} 10 \mathrm{Gd}}, M_{20 \mathrm{Fe}}$, and $M_{10 \mathrm{Fe}}$, respectively. The value of $M_{20 \mathrm{Fe}}$ is comparable with that of $M_{10 \mathrm{Fe}}$ although the $20 \mathrm{Fe}$ glass has $\mathrm{Fe}$ ions twice as many as the $10 \mathrm{Fe}$ glass. In the $20 \mathrm{Fe}$ glass, the magnetization is more suppressed than in the $10 \mathrm{Fe}$ glass by the stronger AFM interactions between the Fe ions. The values of $M_{20 \mathrm{Fe} 20 \mathrm{Gd}}$ and $M_{20 \mathrm{Fe} 10 \mathrm{Gd}}$ are much larger than $M_{20 \mathrm{Fe}}$ because of the large contribution of $\mathrm{Gd}^{3+}$ magnetic moments to the magnetizations. On the other hand, $M_{20 \mathrm{Fe} 20 \mathrm{Gd}}$ is smaller than $M_{20 \mathrm{Gd}}$ although the 20Fe20Gd glass has additional Fe magnetic moments compared to the $20 \mathrm{Gd}$ glass. This result is in accordance with the behavior of $\chi(T)$ shown in Fig. 3(a). The molecular field produced by the SG freezing of the $\mathrm{Fe}$ magnetic moments interplays with the moments of $\mathrm{Gd}$ ions, leading to the significant suppression of magnetization.

To give an insight into the magnetic interactions among the $\mathrm{Fe}$ and $\mathrm{Gd}$ ions, the magnetization curves were analyzed as follows: $M_{20 \mathrm{Fe} 20 \mathrm{Gd}}, M_{20 \mathrm{Fe}}+M_{20 \mathrm{Gd}}, M_{20 \mathrm{Fe} 10 \mathrm{Gd}}$, and $M_{20 \mathrm{Fe}}$ $+\frac{1}{2} M_{20 \mathrm{Gd}}$ are plotted as a function of magnetic field in Fig. $5(\mathrm{~b})$, and $M_{20 \mathrm{Fe} 10 \mathrm{Gd}}, \frac{1}{2} M_{20 \mathrm{Fe}}+\frac{1}{2} M_{20 \mathrm{Fe} 20 \mathrm{Gd}}, M_{20 \mathrm{Fe} 10 \mathrm{~Tb}}$ and $\frac{1}{2} M_{20 \mathrm{Fe}}+\frac{1}{2} M_{20 \mathrm{Fe} 20 \mathrm{~Tb}}$ in Fig. $5(\mathrm{c})$. It is found that $M_{20 \mathrm{Fe}}+M_{20 \mathrm{Gd}}$ and $M_{20 \mathrm{Fe}}+\frac{1}{2} M_{20 \mathrm{Gd}}$ do not coincide with $M_{20 \mathrm{Fe} 20 \mathrm{Gd}}$ and $M_{20 \mathrm{Fe} 10 \mathrm{Gd}}$, respectively. This is attributed to the significant AFM interactions between the Fe and Gd ions, as also discussed in Sec III B. In contrast, $\frac{1}{2} M_{20 \mathrm{Fe} 20 \mathrm{Gd}}+\frac{1}{2} M_{20 \mathrm{Fe}}$ 
coincides well with $M_{20 \mathrm{Fe} 10 \mathrm{Gd}}$. These results indicate that the magnetic interaction between the Gd ions is negligibly small compared to those between the Fe ions and those between the $\mathrm{Fe}$ and $\mathrm{Gd}$ ions. A comparison of these two cases is schematically illustrated in Fig. 5(d); the left and right panels of the Fig. $5(\mathrm{~d})$ correspond to $\frac{1}{2} M_{20 \mathrm{Fe} 20 \mathrm{Gd}}+$ $\frac{1}{2} M_{20 \mathrm{Fe}}$ and $M_{20 \mathrm{Fe} 10 \mathrm{Gd}}$, respectively. The total number of $\mathrm{Fe}$ and $\mathrm{Gd}$ ions is identical to each other, and the Fe ions are homogeneously distributed in both cases. On the other hand, in the left case, all the Gd ions are concentrated in the below half space. Assuming that a magnetic ion only interacts with the neighboring magnetic ions, the number of interacting $\mathrm{Fe}-\mathrm{Fe}$ and Fe-Gd pairs in the left case is the same as that in the right case because of the same concentration of $\mathrm{Fe}$ ions. The most striking difference between the two cases is the number of interacting $\mathrm{Gd}$ pairs. Therefore, in view of the fact that $\frac{1}{2} M_{20 \mathrm{Fe} 20 \mathrm{Gd}}+\frac{1}{2} M_{20 \mathrm{Fe}}$ is almost equal to $M_{20 \mathrm{Fe} 10 \mathrm{Gd}}$, the magnetic interaction between the Gd ions is negligibly small compared to those between the Fe ions and those between the $\mathrm{Fe}$ and $\mathrm{Gd}$ ions. In addition, this is case with $R=\mathrm{Tb}$ as shown in Fig. 5(c); $\frac{1}{2} M_{20 \mathrm{Fe} 20 \mathrm{~Tb}}+\frac{1}{2} M_{20 \mathrm{Fe}}$ merges well with $M_{20 \mathrm{Fe} 10 \mathrm{~Tb}}$, indicating that the magnetic interaction between the $\mathrm{Tb}$ ions is also of little significance.

\section{Temperature dependence of ac magnetic susceptibility}

The temperature dependence of ac magnetic susceptibility is shown for 20Gd glass in Figs. 6(a) and 6(b), for 20Fe glass in Figs. 6(c) and 6(d), and for 20Fe20Gd glass in Figs. 6(e) and 6(f). The amplitude of ac magnetic field was kept at 3 Oe. The frequency, $f$, is $1,10,100$, and $1000 \mathrm{~Hz}$ for the 20Gd glass and $0.3,1,3,10,30,100,300$, and 1000 $\mathrm{Hz}$ for the $20 \mathrm{Fe}$ and $20 \mathrm{Fe} 20 \mathrm{Gd}$ glasses. The real part of ac susceptibility, $\chi^{\prime}(T)$, is almost independent of frequency, $f$, for the 20Gd glass, as depicted in Fig. 6(a), indicating that the present glass is PM down to $2.5 \mathrm{~K}$. This is consistent with the $\chi(T)$ curve shown in Fig. 3(a). In contrast, the imaginary part of ac susceptibility, $\chi^{\prime \prime}(T)$, exhibits a weak $f$ dependence, which is reminiscent of some magnetic transition at lower temperatures.
For the 20Fe glass, both $\chi^{\prime}(T)$ and $\chi^{\prime \prime}(T)$ exhibit an $f$ dependence as observed in SGs; they show a peak and the peak temperature shifts to a higher-temperature side with an increase in $f$. According to the dynamic scaling hypothesis, provided that this system exhibits a conventional critical slowing down toward the critical temperature $T_{c}$, the temperature variation in relaxation time, $\tau$, is described by

$$
\tau=\tau_{0}\left(\frac{T-T_{c}}{T_{c}}\right)^{-z v},
$$

where $z v$ is the dynamic exponent and $\tau_{0}$ is the microscopic relaxation time. ${ }^{30}$ The temperature dependence of $\tau$ can be derived from the data of ac susceptibility; $\tau$ becomes $1 / f$ at the peak temperature of $\chi^{\prime}(T)$ measured by ac magnetic field with frequency $f$. Since the peak of $\chi^{\prime}(T)$ is very broad in this case, we employed the inflection temperature of $\chi^{\prime \prime}(T)$ instead of the peak temperature of $\chi^{\prime}(T) .{ }^{30-32}$ The best fitting of Eq. (11) to the experimental data gives $z v=9.4, T_{c}=3.6 \mathrm{~K}$ and $\tau_{0}=1.2 \times 10^{-6} \mathrm{~s}$ [see the inset of Fig. 6(c)]. The value of $z v$ is comparable with those reported for conventional SGs, including amorphous oxide SGs. ${ }^{11,17,30,33}$ The value of $\tau_{0}$ is much larger than the typical values for atomic SGs, where $\tau_{0} \sim 10^{-13}$ s, i.e., the spin-flip time of individual magnetic moments belonging to atoms or ions, but similar to those reported for strongly interacting nanoparticle systems and superspin glasses, for which $\tau_{0} \sim 10^{-6} \mathrm{~s} .{ }^{34,35}$ This is consistent with the bifurcation of $\chi_{\mathrm{ZFC}}(T)$ and $\chi_{\mathrm{FC}}(T)$ above the peak temperature of $\chi_{\mathrm{ZFC}}(T)$ discussed in Sec. III B, indicating that magnetic clusters consist of some of the Fe ions in the present glass. These results imply the SG freezing of the magnetic clusters in the present glass.

Both $\chi^{\prime}(T)$ and $\chi^{\prime \prime}(T)$ for the 20Fe20Gd glass also strongly depend on $f$, although $\chi^{\prime}(T)$ increases monotonously with a decrease in temperature. At low temperatures, $\chi^{\prime}(T)$ grows lower as $f$ becomes higher, being consistent with the behavior of conventional SGs. Also, $\chi^{\prime \prime}(T)$ exhibits behavior similar to the $20 \mathrm{Fe}$ glass; the maximum temperature of $\chi^{\prime \prime}(T)$ shifts to a higher-temperature side with an increase in $f$. Utilizing the inflection temperature of the $\chi^{\prime \prime}(T)$ curve, the temperature dependence of $\tau$ was analyzed through Eq. (11), leading to
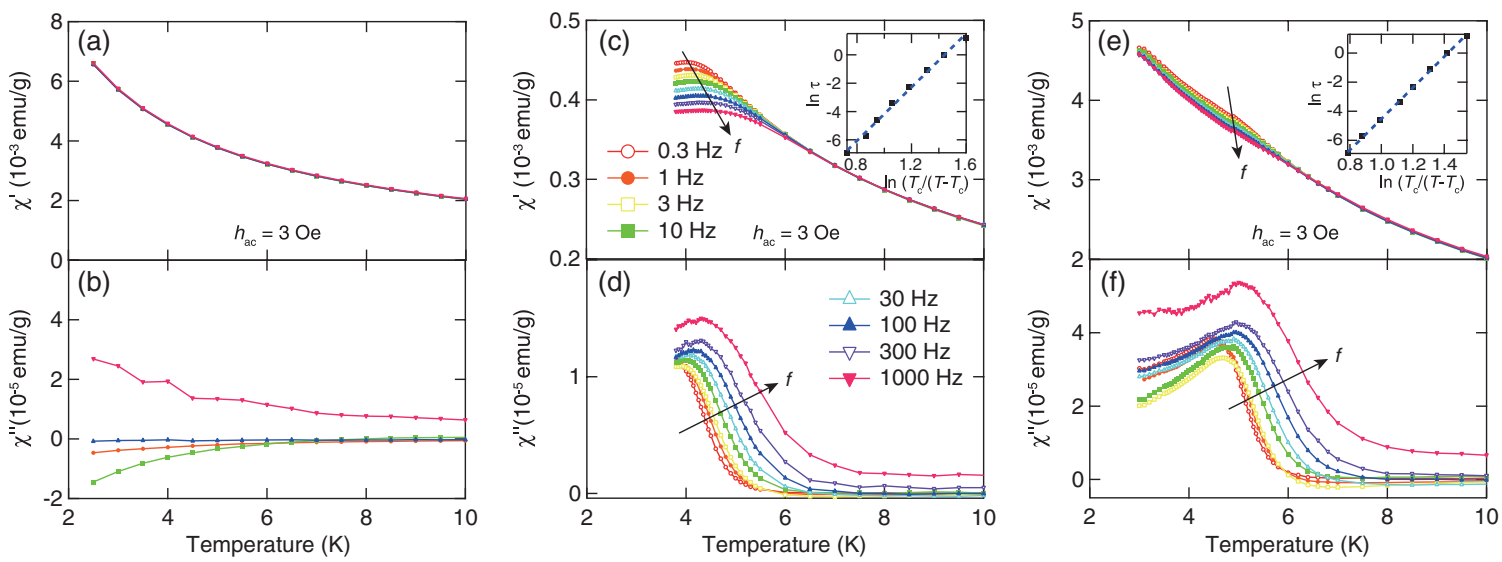

FIG. 6. (Color online) Temperature dependence of real and imaginary parts of ac magnetic susceptibility, $\chi^{\prime}(T)$ and $\chi^{\prime \prime}(T)$, respectively, for (a) and (b) 20Gd, (c) and (d) 20Fe, and (e) and (f) 20Fe20Gd glasses. The amplitude of ac magnetic field was kept at 3 Oe. The frequency, $f$, is $1,10,100$, and $1000 \mathrm{~Hz}$ for $20 \mathrm{Gd}$, and $0.3,1,3,10,30,100,300$, and $1000 \mathrm{~Hz}$ for $20 \mathrm{Fe}$ and $20 \mathrm{Fe} 20 \mathrm{Gd}$. 
$z v=10.7, T_{c}=4.2 \mathrm{~K}$, and $\tau_{0}=2.4 \times 10^{-6} \mathrm{~s}$ [see the inset of Fig. 6(e)]. The values of $z v$ and $\tau_{0}$ are comparable with those obtained for the $20 \mathrm{Fe}$ glass, evidencing the SG nature similar to that in the $20 \mathrm{Fe}$ glass.

The relative shift of $T_{f}$ per decade of $f, \Phi=$ $\Delta T_{f} /\left(T_{f} \Delta \log f\right)$, provides criteria for distinguishing SG freezing from superparamagnetic relaxation, i.e., the thermal relaxation process of noninteracting nanoparticles or clusters. The values of $\Phi$ are 0.060 and 0.052 for the $20 \mathrm{Fe}$ and 20Fe20Gd glasses, respectively. These values are slightly larger than those reported for canonical SGs, such as $C u \mathrm{Mn}$ ( $\Phi=0.005)$ and $A u \mathrm{Fe}(0.010)$, and comparable to cobalt aluminosilicate glasses (0.06). ${ }^{1}$ Although the values of $\Phi$ are significantly smaller than those reported for superparamagnets $\left(\Phi>10^{-1}\right),{ }^{1}$ the contribution of the thermal relaxation of magnetic clusters to the magnetic transitions cannot be excluded. At around the spin-freezing temperatures, the ac susceptibility measurements probably capture the superparamagnetic blocking of some magnetic clusters composed of the $\mathrm{Fe}$ ions due to their magnetic anisotoropy energies as well as the critical slowing down due to the SG transitions. Such complex behavior may cause rather large value of $\tau_{0}$ for the present glasses.

\section{E. ZFC memory effect}

The magnetic aging and memory effect measurements have been performed in order to corroborate the SG nature of the present glasses. The magnetic aging effects peculiar to SGs have been recently investigated by a variety of methods for various SG systems. ${ }^{11,36-40}$ Here, we used the genuine ZFC protocol proposed by Mathieu et al. ${ }^{37}$ The $20 \mathrm{Fe}$ glass was cooled at a rate of $0.1 \mathrm{~K} / \mathrm{min}$ in the absence of magnetic field from a temperature higher than $T_{f}=4.5 \mathrm{~K}$ with an intermittent stop at $3 \mathrm{~K}$ for $3 \mathrm{~h}$. Then, the $\chi_{\mathrm{s}}(T)$ curve was recorded during subsequent heating at a rate of $0.1 \mathrm{~K} / \mathrm{min}$ under a measuring magnetic field of 500 Oe. The results are shown in Fig. 7. The $\chi_{\mathrm{s}}(T)$ curve takes smaller values at around $3 \mathrm{~K}$ compared to the $\chi_{\text {ref }}(T)$ curve, which was obtained by the conventional ZFC process without intermittent stops. The difference $\Delta \chi(T)=$ $\chi_{\mathrm{s}}(T)-\chi_{\mathrm{ref}}(T)$ clearly manifests a so-called memory dip at around $3 \mathrm{~K}$. Sasaki et al. ${ }^{40}$ demonstrated theoretically and experimentally that a memory is imprinted during the aging in the absence of magnetic field only for SGs and strongly interacting nanoparticles systems or superspin glasses but not for noninteracting superparamagnets. Hence, the observation of the ZFC memory effect warrants the cooperative freezing of the magnetic moments, i.e., SG and/or superspin-glass transition.

\section{F. Magnetic relaxation under magnetic field}

The magnetic relaxation effects under a magnetic field, which originates from the slow spin dynamics of SGs, was investigated for $20 \mathrm{Fe}, 20 \mathrm{Fe} 20 \mathrm{Gd}, 20 \mathrm{Fe} 10 \mathrm{Gd}, 20 \mathrm{Fe} 20 \mathrm{~Tb}$, and $20 \mathrm{Fe} 10 \mathrm{~Tb}$ glasses. The samples were cooled to $2 \mathrm{~K}$ at a rate of $1.0 \mathrm{~K} / \mathrm{min}$ in the absence of magnetic field, and the time evolution of magnetization was measured at $2 \mathrm{~K}$ under a magnetic field of 50 Oe.

The change of magnetization as a function of time, $\Delta M=$ $M(t)-M(0)$, is shown in Fig. 8. It is found that $\Delta M$ is

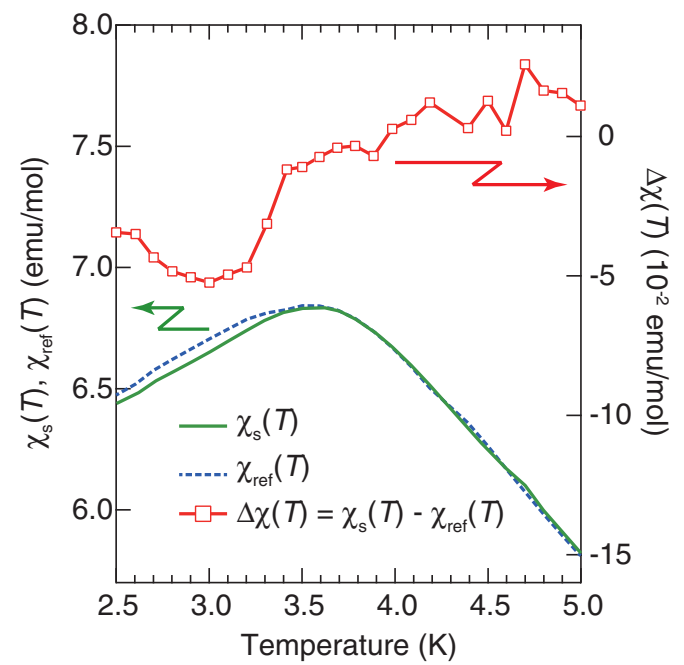

FIG. 7. (Color online) Temperature dependence of dc susceptibility for the $20 \mathrm{Fe}$ glass measured on heating after ZFC with and without an intermittent stop at $3 \mathrm{~K}$ for $3 \mathrm{~h} . \chi_{\mathrm{s}}(T)$ and $\chi_{\text {ref }}(T)$ are the $\mathrm{dc}$ susceptibilities with and without the intermittent stop, respectively. The difference between $\chi_{\mathrm{s}}(T)$ and $\chi_{\mathrm{ref}}(T), \Delta \chi(T)$, is also illustrated.

proportional to the logarithm of time for the present glasses, which is characteristic of SGs. ${ }^{1}$ It is notable that the higher the contents of $\mathrm{Gd}$ and $\mathrm{Tb}$ ions, the larger the rate of increase in magnetization becomes, although the SG states mainly stem from spin freezing of the Fe ions. It is considered that the magnetization due to PM Gd and $\mathrm{Tb}$ moments becomes larger because of the time evolution of the molecular field accompanied by relaxation of the Fe moments, although we cannot completely rule out that this is due to relaxation of the $\mathrm{Gd}$ and $\mathrm{Tb}$ moments which freeze into the SG states. Both possibilities clearly demonstrate the importance of the magnetic interactions between the $\mathrm{Fe}$ and $\mathrm{RE}$ ions for the magnetic properties of the present glasses.

\section{SUMMARY}

We have systematically investigated the magnetic properties of aluminoborate glasses with the composition

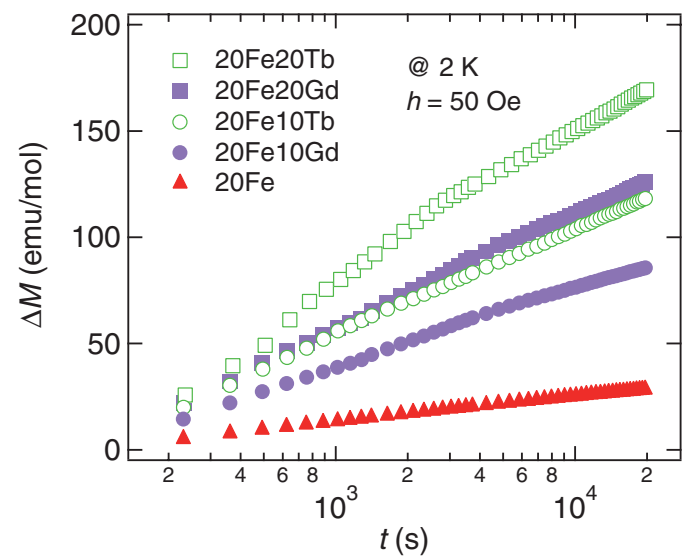

FIG. 8. (Color online) Time dependence of magnetization measured under a magnetic field of 50 Oe at $2 \mathrm{~K}$ for $20 \mathrm{Fe}, 20 \mathrm{Fe} 20 \mathrm{Gd}$, $20 \mathrm{Fe} 10 \mathrm{Gd}, 20 \mathrm{Fe} 20 \mathrm{~Tb}$, and $20 \mathrm{Fe} 10 \mathrm{~Tb}$ glasses. 
(mol\%) of $x \mathrm{Fe}_{2} \mathrm{O}_{3} \cdot(20-x) \mathrm{Ga}_{2} \mathrm{O}_{3} \cdot y R_{2} \mathrm{O}_{3} \cdot(20-y) \mathrm{La}_{2} \mathrm{O}_{3} \cdot 3 \mathrm{Al}_{2}$ $\mathrm{O}_{3} \cdot 57 \mathrm{~B}_{2} \mathrm{O}_{3} \quad(x=0,15$, and 20 , and $y=0,10$, and 20), where $R=\mathrm{Sm}, \mathrm{Gd}$, and $\mathrm{Tb}$, to understand the magnetic interactions among the $\mathrm{Fe}$ and $\mathrm{RE}$ ions and the magnetic structures. The glasses with $x=20$ exhibit SG transition at low temperatures as revealed by the temperature dependence of $\mathrm{dc}$ and ac susceptibilities as well as the magnetic aging memory effect, indicating that the magnetic interactions between the $\mathrm{Fe}$ ions are the most responsible for the SG transitions. The spin-freezing temperatures become higher with an increase in the content of RE ions in the case of $R=\mathrm{Gd}$ and $\mathrm{Tb}$. Thus, the magnetic interactions between the $\mathrm{Fe}$ and $\mathrm{RE}$ ions are also important for the SG transitions. The magnetization of RE ions is suppressed by the AFM interactions between the Fe and RE ions, i.e., the molecular field generated by the Fe ions. The detailed analysis of the dc susceptibility based on the molecular-field theory as well as of the magnetic-field dependence of magnetization supports the idea that the magnetic interactions between the

*tanaka@dipole7.kyoto-u.ac.jp

${ }^{1}$ J. A. Mydosh, Spin Glasses: An Experimental Introduction (Taylor \& Francis, London, 1993).

${ }^{2}$ R. A. Verhelst, R. W. Kline, A. M. de Graat, and H. O. Hooper, Phys. Rev. B 11, 4427 (1975).

${ }^{3}$ F. Wedgwood and A. Wright, J. Non-Cryst. Solids 21, 95 (1976).

${ }^{4}$ J. Ferre, J. Pommier, J. Renard, and K. Knorr, J. Phys. C 13, 3697 (1980).

${ }^{5}$ C. Dupas, J.-P. Renard, G. Fonteneau, and J. Lucas, J. Magn. Magn. Mater. 27, 152 (1982).

${ }^{6}$ A. Ito, E. Torikai, H. Yamauchi, and Y. Syono, J. Phys. C 15, 2759 (1982).

${ }^{7}$ P. Beauvillain, C. Dupas, J. P. Renard, and P. Veillet, Phys. Rev. B 29, 4086 (1984).

${ }^{8}$ J. P. Sanchez, J. M. Friedt, R. Horne, and A. J. V. Duyneveldt, J. Phys. C 17, 127 (1984).

${ }^{9}$ S. Nakamura, S. Soeya, N. Ikeda, and M. Tanaka, J. Appl. Phys. 74, 5652 (1993).

${ }^{10}$ J. L. Shaw, A. C. Wright, R. N. Sinclair, G. K. Marasinghe, D. Holland, M. R. Lees, and C. R. Scales, J. Non-Cryst. Solids 345-346, 245 (2004).

${ }^{11}$ H. Akamatsu, K. Tanaka, K. Fujita, and S. Murai, Phys. Rev. B 74, 012411 (2006).

${ }^{12}$ H. Akamatsu, K. Tanaka, K. Fujita, and S. Murai, J. Magn. Magn. Mater. 310, 1506 (2007).

${ }^{13}$ H. Akamatsu, K. Tanaka, K. Fujita, and S. Murai, J. Phys. Condens. Matter 20, 235216 (2008).

${ }^{14}$ H. Akamatsu, K. Fujita, S. Murai, and K. Tanaka, Appl. Phys. Lett. 92, 251908 (2008).

${ }^{15}$ K. Tanaka, H. Akamatsu, S. Nakashima, and K. Fujita, J. Non-Cryst. Solids 354, 1347 (2008).

${ }^{16}$ H. Akamatsu, S. Murai, K. Fujita, and K. Tanaka, Adv. Mater. Res. 39-40, 207 (2008).

${ }^{17}$ H. Akamatsu, S. Oku, K. Fujita, S. Murai, and K. Tanaka, Phys. Rev. B 80, 134408 (2009).

${ }^{18}$ G. C. Lau, T. Klimczuk, F. Ronning, T. M. McQueen, and R. J. Cava, Phys. Rev. B 80, 214414 (2009).

${ }^{19}$ J. Schoenes, E. Kaldis, W. Thöni, and P. Wachter, Phys. Sta. Sol. (a) 51, 173 (1979).
Fe and RE ions as well as those between the Fe ions play a significant role in the magnetic properties of the present glasses while those between the RE ions are of little importance. Also, the relaxation of magnetization under a manetic field is strongly affected by the magnetic interactions between the $\mathrm{Fe}$ and RE ions. Thus, the magnetic interactions between the $\mathrm{Fe}$ and $\mathrm{RE}$ ions are responsible for their intriguing magnetic properties, similarly to RE garnet ferrites and orthoferrites.

\section{ACKNOWLEDGMENTS}

The authors thank M. Tosaki and Y. Isozumi of Radioisotope Research Center, Kyoto University, for the Mössbauer effect measurements. This research was partially supported by the MEXT Grant-in-Aid for Scientific Research (B)(No. 22360273) and Challenging Exploratory Research(No. 23655198). One of the authors (H.A.) thanks the JSPS for Grant No. 22-1280.

${ }^{20}$ H. Akamatsu, K. Fujita, S. Murai, and K. Tanaka, Phys. Rev. B 81, 014423 (2010)

${ }^{21}$ Y. Zong, K. Kugimiya, K. Fujita, H. Akamatsu, K. Hirao, and K. Tanaka, J. Non-Cryst. Solids 356, 2389 (2010).

${ }^{22}$ H. Akamatsu, K. Fujita, Y. Zong, N. Takemoto, S. Murai, and K. Tanaka, Phys. Rev. B 82, 224403 (2010).

${ }^{23}$ Y. Zong, K. Fujita, H. Akamatsu, S. Murai, and K. Tanaka, Phys. Stat. Sol. (c), in press (2011).

${ }^{24}$ C. Chappert, P. Beauvillain, J. P. Renard, and K. Knorr, Solid State Commun. 31, 213 (1979).

${ }^{25}$ K. Sato, K. Yamaguchi, F. Maruyama, and N. Nishi, Phys. Rev. B 63, 104416 (2001).

${ }^{26}$ R. Pauthenet, J. Appl. Phys. 29, 253 (1958).

${ }^{27}$ Y. Tokunaga, N. Furukawa, H. Sakai, Y. Taguchi, T.-h. Arima, and Y. Tokura, Nat. Mater. 8, 558 (2009).

${ }^{28}$ D. Virgo and B. Mysen, Phys. Chem. Minerals 12, 65 (1985).

${ }^{29}$ S. Nagata, P. H. Keesom, and H. R. Harrison, Phys. Rev. B 19, 1633 (1979).

${ }^{30}$ K. Gunnarsson, P. Svedlindh, P. Nordblad, L. Lundgren, H. Aruga, and A. Ito, Phys. Rev. Lett. 61, 754 (1988).

${ }^{31}$ N. Bontemps, J. Rajchenbach, R. V. Chamberlin, and R. Orbach, Phys. Rev. B 30, 6514 (1984).

${ }^{32}$ E. Vincent, J. Hammann, and M. Alba, Solid State Commun. 58, 57 (1986)

${ }^{33}$ P. Nordblad, J. Phys. Condens. Matter 16, S715 (2004).

${ }^{34}$ C. Djurberg, P. Svedlindh, P. Nordblad, M. F. Hansen, F. Bødker, and S. Mørup, Phys. Rev. Lett. 79, 5154 (1997).

${ }^{35}$ M. F. Hansen, P. E. Jnsson, P. Nordblad, and P. Svedlindh, J. Phys. Condens. Matter 14, 4901 (2002).

${ }^{36}$ K. Jonason, E. Vincent, J. Hammann, J. P. Bouchaud, and P. Nordblad, Phys. Rev. Lett. 81, 3243 (1998).

${ }^{37}$ R. Mathieu, P. Jönsson, D. N. H. Nam, and P. Nordblad, Phys. Rev. B 63, 092401 (2001).

${ }^{38}$ V. Dupuis, E. Vincent, J.-P. Bouchaud, J. Hammann, A. Ito, and H. A. Katori, Phys. Rev. B 64, 174204 (2001).

${ }^{39}$ S. Sahoo, O. Petracic, W. Kleemann, P. Nordblad, S. Cardoso, and P. P. Freitas, Phys. Rev. B 67, 214422 (2003).

${ }^{40}$ M. Sasaki, P. E. Jönsson, H. Takayama, and H. Mamiya, Phys. Rev. B 71, 104405 (2005). 